\title{
A GENERAL FORMULATION OF THE BOUNDARY CONDITIONS ON THE VECTOR POTENTIAL IN THREE-DIMENSIONAL HYDRODYNAMICS*
}

\author{
BY \\ G. J. HIRASAKI AND J. D. HELLUMS \\ Rice University
}

1. Introduction. In recent years the mathematical formulation and solution of problems involving three-dimensional fluid motion has become of increasing interest. One of the most promising ways of expressing the equations of incompressible fluid motion in a form suitable for solution by digital computation involves the use of a vector potential. The purpose of this paper is to discuss the use of the vector potential and to give the most general form of the boundary conditions for an incompressible flow in which the velocity is specified on the boundaries of the region. Both finite regions and infinite regions with the velocity regular at infinity are considered.

The use of the vector potential to calculate the velocity from a given vorticity field has been discussed by numerous workers starting with Lamb [1]. A survey of articles on the vector potential is given in the book by Jacob [2]. The problem of current interest is solution of the equations of motion so the vorticity field as well as the velocity is to be determined rather than being specified. Surprisingly enough, the hydrodynamics literature on this important problem is extremely sparse in contrast to that on related problems in electromagnetic field theory. Apparently only two recent papers refer to the boundary conditions on the vector potential. Timman [3] suggested that the vector potential could be required to vanish on solid surfaces. Moreau [4] pointed out that Timman's conditions were incorrect and that when the velocity is tangential to the boundaries the vector potential is normal to the boundaries. Aziz and Hellums [5] used the vector potential in numerical solutions of the three-dimensional equations of motion in transient, laminar natural convection.

In $\$ 2$ we discuss the formulation of the equations of motion in terms of the vorticity and a vector potential. In such problems the velocity is regarded as specified on the boundaries. Hence, we consider the problem of finding boundary conditions on the vector potential which imply the specified velocity distribution.

In $\$ 3$ the hypotheses on the region and the velocity field are stated and certain properties of the vector potential are discussed. In $\$ 4$ a vector $\mathbf{B}$ normal to the boundaries is introduced. We show that $\mathbf{B}$ satisfies a certain second order differential equation and that finding $\mathbf{B}$ is equivalent to finding the desired boundary conditions. In $\$ 5$ the procedure for finding the boundary condition on the normal component of the vector potential is discussed. The boundary conditions on the vorticity at a free surface or at an interface between two viscous fluids are given in $\$ 6$. Finally, in $\$ 7$ the equations are given for several important special cases.

2. Hydrodynamic equations and problem formulation. The hydrodynamic equations in a form convenient for solution by numerical methods are given below.

*Received April 18, 1967. 


$$
\begin{gathered}
\partial \mathrm{W} / \partial t+\mathrm{V} \cdot \nabla \mathrm{W}-\mathrm{W} \cdot \nabla \mathrm{V}=\nabla \times \mathrm{F} / \rho+\nu \nabla^{2} \mathrm{~W}, \\
\nabla^{2} \mathrm{~A}=-\mathrm{W},
\end{gathered}
$$

where $\mathbf{A}$ is the vector potential, $\mathbf{W}$ is the vorticity, $\mathbf{V}$ is the velocity, $\mathbf{F}$ is a nonconservative body force, $\rho$ is the density, and $\nu$ is the kinematic viscosity. Equation (2.1) is the vorticity transfer equation for an incompressible Newtonian fluid and (2.2) will be discussed below. The vector potential and vorticity are defined by (2.3) and (2.4) respectively.

$$
\begin{aligned}
& \mathbf{V}=\nabla \times \mathbf{A}, \\
& \mathbf{W}=\nabla \times \mathbf{V} .
\end{aligned}
$$

Since the curl of the gradient of a scalar is identically zero, $\mathbf{A}$ is arbitrary to a gradient of a scalar. The continuity equation for an incompressible fluid is identically satisfied by (2.3).

Substituting (2.3) into (2.4) we have

$$
\mathbf{W}=\nabla \times(\nabla \times \mathbf{A}) \equiv \nabla(\nabla \cdot \mathbf{A})-\nabla^{2} \mathbf{A}
$$

Equation (2.5) reduces to (2.2) if $\mathbf{A}$ is solenoidal, and a solenoidal $\mathbf{A}$ can always be found by the Gauge transformation.

Equations (2.1), (2.2), and (2.3) together with the specification of $\mathrm{V}$ on the boundaries constitute a complete problem which presumably determines the velocity distribution. For purposes of solving the equations it is necessary to express the boundary conditions in terms of the vector potential and vorticity. In numerical work an iterative procedure is used in which the two equations are treated within a given iteration as if uncoupled with the coupling introduced between subsequent iterations. Hence, the conditions on $\mathbf{A}$ are desired which imply the desired conditions on V. Finding these conditions for arbitrary $\mathrm{V}$ is the subject of this paper.

3. Hypothesis and preliminary results. Iet the region of space for which we wish to determine the flow field be denoted by $V$ and let it be a regular or an infinite regular region of space as defined by Kellogg [6]. Iet $S$ be the surface(s) that is (are) the bound$\operatorname{ary}(\mathrm{ies})$ of $V$. To consider the possibility of multiple disconnected surfaces denote $S$ by $S=\bigcup_{i=1}^{v} S_{i}$ where each $S_{i}$ is regular, closed, and simply connected. Also denote the surface integral over $S$ to mean $\int_{S}=\sum_{i=1}^{N} \oint_{s_{i}}$. The net flux across each of the surfaces $S_{i}$ must be equal to zero, i.e.,

$$
\oint_{s_{i}} \mathbf{n} \cdot \mathrm{V} d a=0, \quad i=1, \cdots, N
$$

For the purpose of this paper we will have to require more smoothness on $S$ than that required by Kellogg. Let $y^{3}=f\left(y^{1}, y^{2}\right)$ be a standard representation for a regular surface element of $S$. Define a "face" to be a region of $S$ such that the third derivative of $f\left(y^{1}, y^{2}\right)$ satisfies a uniform Hölder condition [6]. $S$ must consist of a finite number of faces as defined here and each face must be bounded by a finite number of regular ares.

Let the velocity, $\mathrm{V}$, have continuous derivatives of the second order in the interior of $V$, continuous one-sided derivatives normal to $S$ on $S$ and continuous first derivatives with respect to the surface coordinates on $S$. The velocity field is solenoidal, $\nabla \cdot V=0$, since the flow is incompressible. If the region is infinite, $\mathrm{V}$ must be regular at infinity [7]. 
Theorem 3.1. Given a vector $\mathrm{V}$ with the above conditions in the region $\mathrm{V}$, there exists a vector potential $\mathbf{A}$ such that $\mathbf{V}=\nabla \times \mathbf{A}$ and $\nabla \cdot \mathbf{A}=0$ [8].

Lemмa 3.1. Let $\mathrm{E}$ be continuously differentiable on each face of $S_{i}$, have a continuous tangential component across an edge, and satisfy the equation

$$
\mathbf{n} \cdot \nabla \times \mathbf{E}=\mathbf{0}
$$

on each face. Then the tangential projection of $\mathbf{E}$ on each face of $S_{i} i$ s

$$
\mathrm{E}_{T}=\nabla_{s} \psi
$$

where

$$
\psi(P)=\int_{P_{0}}^{P} \mathbf{E} \cdot d \mathbf{X}
$$

The line integral is taken on $S_{i}$ where $P_{0}$ is an arbitrary point on $S_{i}$.

Theorem 3.2. Let $\mathbf{A}_{s}$ be a vector tangent to $S$ with continuous tangential values across an edge, continuously differentiable on each face of $S$ and satisfying

$$
\mathrm{n} \cdot \nabla, \times \mathbf{A}_{\mathbf{s}}=\mathrm{n} \cdot \mathrm{V} .
$$

Then there exists a vector potential, $\mathbf{A}^{\prime}$, having the same divergence and curl as $\mathbf{A}$ and having A, as its tangential projection on $S$.

Proof. The original vector potential, A, also satisfies (3.1) on $S$ since

$$
\mathbf{n} \cdot \nabla \cdot \times \mathbf{A} \equiv \mathbf{n} \cdot \nabla \times \mathbf{A}=\mathbf{n} \cdot \mathbf{V} .
$$

Thus

$$
\mathrm{n} \cdot \nabla_{\mathrm{s}} \times\left(\mathbf{A}-\mathbf{A}_{\mathrm{s}}\right)=0 .
$$

We may now express the tangential projection of $\mathbf{A}-\mathbf{A}_{\boldsymbol{s}}$ as

$$
\left(\mathbf{A}-\mathbf{A}_{s}\right)_{T}=A_{T}-A_{s}=\nabla_{s} \psi_{i}
$$

where

$$
\psi_{i}(P)=\int_{P_{0}}^{P}\left(\mathbf{A}-\mathbf{A}_{s}\right) \cdot d \mathbf{X} .
$$

$\mathbf{A}_{\boldsymbol{T}}$ is the tangential projection of $\mathbf{A}$ on $S$ and the liine integral is evaluated on $S_{i}$ with $P_{0}$ as an arbitrary point on $S_{i} . \nabla_{s} \psi_{i}$ is unique but $\psi_{i}$ is arbitrary by a constant which depends on the location of $P_{0}$.

Let $\phi$ be a function harmonic in $V$ and having its value equal to $\psi_{i}$ on $S_{i}$. The existence of $\phi$ is established by the Dirichlet problem. If $S$ is a single connected surface then $\phi$ is arbitrary by a constant but $\nabla \phi$ is unique. However, if $S$ is a sum of multiple disconnected surfaces, then neither $\phi$ nor $\nabla \phi$ is unique. Let

$$
\mathbf{A}^{\prime}=\mathbf{A}-\nabla \phi .
$$

Then on $S$

$$
\begin{aligned}
\mathbf{A}_{T}^{\prime} & =\mathbf{A}_{T}-\nabla_{s} \boldsymbol{\phi} \\
& =\mathbf{A}_{T}-\nabla_{s} \psi_{i} \\
& =\mathbf{A}_{s} .
\end{aligned}
$$


The divergence and curl of $\mathbf{A}^{\prime}$ are equal to that of $\mathbf{A}$ since

$$
\begin{aligned}
\nabla \cdot \nabla \phi & \equiv \nabla^{2} \phi=0, \\
\nabla \times \nabla \phi & \equiv 0 .
\end{aligned}
$$

We have proven that $\mathbf{A}^{\prime}$ has the same divergence and curl as $\mathbf{A}$ so it may replace $\mathbf{A}$ in the differential equation (2.2). $\mathbf{A}^{\prime}$ is a vector with $\mathbf{A}_{s}$ as its tangential projection on $S$.

\section{Formulation as a differential equation in a normal vector $B$.}

Lemma 4.1. Let B be a vector normal to $S$ and satisfying the differential equation

$$
\mathbf{n} \cdot \nabla, \times(\nabla, \times \mathbf{B})=\mathbf{n} \cdot \mathbf{V}
$$

on each face. Let

$$
\mathbf{A}_{s}=\nabla, \times \mathbf{B} .
$$

Then $\mathbf{A}_{\mathrm{s}}$ satisfies (3.1) on each face and is tangential to each face.

The surface curl of a spatial vector may be expressed in the tensor notation such as that used by Aris [9].

$$
(\nabla . \times \mathrm{B})_{i}=\epsilon_{i j k} a^{\alpha \beta} t_{\beta}^{i} B_{, \alpha}^{k} .
$$

Equation (4.1) may be written in terms of the components of $B$ by two applications of the surface curl operator and the scalar product with the normal vector. After some simplification the results are

$$
\begin{aligned}
\mathbf{n} \cdot \nabla_{\bullet} \times(\nabla, \times \mathbf{B}) & =n_{j} a^{\gamma \rho} a^{\alpha \beta} t_{\rho}^{m} t_{\beta, \gamma}^{j} g_{k m} B_{, \alpha}^{k}-n_{k} a^{\gamma \alpha} B_{, \alpha \gamma}^{k} \\
& =n_{j} V^{j} .
\end{aligned}
$$

Since $B^{k}$ is normal to $S$, let

$$
\begin{gathered}
B^{k}=\left(B^{r} n_{r}\right) n^{k}, \\
B_{. \alpha}^{k}=\frac{\partial\left(B^{r} n_{r}\right) n^{k}}{\partial u^{\alpha}}+\left(B^{r} n_{r}\right) n_{, \alpha}^{k}, \\
g_{k m} t_{\rho}^{m} n^{k}=0, \\
g_{k m} t_{\rho}^{m} n_{, \alpha}^{k}=-b_{\alpha \rho}, \\
n_{j} t_{\beta, \gamma}^{j}=b_{\beta \gamma}, \\
n_{j} a^{\gamma \rho} a^{\alpha \beta} t_{\rho}^{m} t_{\beta, \gamma}^{i} g_{k m} B_{, \alpha}^{k}=-a^{\gamma \rho} a^{\alpha \beta} b_{\alpha \rho} b_{\beta \gamma}\left(B^{r} n_{r}\right)=\left(2 K-4 H^{2}\right)\left(B^{r} n_{r}\right)
\end{gathered}
$$

where $K$ is the Gaussian curvature and $H$ is the mean curvature of the surface.

Therefore

$$
\left(2 K-4 H^{2}\right)\left(B^{r} n_{r}\right)-n_{k} a^{\alpha \gamma} B_{, \alpha \gamma}^{k}=n_{i} V^{i} .
$$

If one of the coordinate surfaces is made to coincide with the face of $S$ and the other two coordinates are orthogonal with the first, $\mathbf{B}$ will have only one nonzero component on that face. Equation (4.5) will then result in a partial differential equation of the second order for the normal component of $\mathbf{B}$. 
The partial differential equation is valid only on the faces of $S$ and boundary conditions must be specified for the normal component of B on the edges or, if there are no edges, on any closed curve on $S_{i}$. The boundary conditions must satisfy two conditions:

(a) the component of $\nabla_{\mathrm{s}} \times \mathrm{B}$ tangential to the edge must be continuous across the edge, and

(b) on each face $\nabla, \times$ B must satisfy

$$
\begin{aligned}
\int_{S^{\prime}} \mathbf{n} \cdot \mathbf{V} d a & =\int_{S^{\prime}} \mathbf{n} \cdot \nabla_{*} \times\left(\nabla_{s} \times \mathbf{B}\right) d a \\
& =\oint_{C^{\prime}}\left(\nabla_{s} \times \mathbf{B}\right) \cdot d \mathbf{X}
\end{aligned}
$$

where $S^{\prime}$ is a face of $S$ and $C^{\prime}$ is the boundary of $S^{\prime}$.

The conditions $(a)$ and $(b)$ are boundary conditions on the derivative of $\mathbf{B}$ normal to an edge or a linear combination of $\mathbf{B}$ and the derivative of $\mathbf{B}$ normal to an edge, i.e. boundary condition of the second or third kind. Condition $(b)$ is a necessary condition for the existence of a solution to (4.5).

In a plane surface with straight edges, condition $(a)$ requires that the derivative of $\mathbf{B}$, normal to the edge, be continuous across the edge and condition (b) requires that

$$
\int_{s} \mathbf{n} \cdot \mathbf{V} d a=-\oint_{C}, \frac{\partial(\mathbf{n} \cdot \mathbf{B})}{\partial n} d s
$$

where $\partial / \partial n$ is the derivative in the direction of the outward normal to the edge. This second condition is a necessary condition for the existence of the Neumann problem for $\mathbf{n} \cdot \mathbf{B}$.

Lemma 4.2. Boundary conditions (a) and (b) can both be satisfied on all of $S$ if and only if $\mathrm{V}$ satisfies

$$
\oint_{S_{i}} \mathbf{n} \cdot \mathrm{V} d a=0, \quad i=1, \cdots, N .
$$

Proof. Stokes' theorem may be applied to $\mathbf{A}_{s}$ over all of $S$ by taking the circuit integrals along both sides of the edges. Because of condition $(a)$ the net sum of the circuit integrals is equal to zero. Since $n \cdot \nabla \cdot \times \mathbf{A}_{s}=\mathrm{n} \cdot \mathrm{V}$, the net sum of the surface integrals is the left side of (4.7). Thus, conditions $(a)$ and $(b)$ can be satisfied on all of $S$ only if (4.7) is satisfied.

If (4.7) is satisfied and the boundary conditions $(a)$ and $(b)$ are evaluated in a sequence of faces such that the faces for which the boundary conditions have not yet been specified are connected then conditions $(a)$ and $(b)$ can be satisfied on all of the faces.

Equation (4.7) has been adopted as a hypothesis in this paper.

LEmma 4.3. Equation (4.5) with only one nonzero component of $\mathbf{B}$ is an elliptic partial differential equation.

Proof. The second order terms of the equation are

$$
n_{k} a^{\alpha \gamma} \partial^{2} B^{k} / \partial u^{\alpha} \partial u^{\gamma}
$$

where $B^{k}$ is nonzero for only one $k$. A necessary and sufficient condition for the equation to be elliptic is that the matrix $\left(a^{\alpha \gamma}\right)$ be positive definite [10]. 
A regular surface element has a standard representation

$$
y^{3}=f\left(y^{1}, y^{2}\right)
$$

where in this case let $u^{1}=y^{1}$ and $u^{2}=y^{2}$. Then

$$
\operatorname{det}\left(a^{\alpha \gamma}\right)=1+\left(\partial f / \partial y^{1}\right)^{2}+\left(\partial f / \partial y^{2}\right)^{2}>0 .
$$

Lемма 4.4. The second order derivatives of every solution to (4.5) satisfy a Hölder condition.

Proof. Equation (4.5) is elliptic by Lemma 4.3. Let $y^{3}=f\left(y^{1}, y^{2}\right)$ be the standard representation of a face of $S$ as before. Derivatives of the third order of $f$ satisfy a Hölder condition by hypothesis and are the highest order that appear in the coefficient in the left side of (4.5). The right side, $\mathbf{n} \cdot \mathbf{V}$, is continuously differentiable by hypothesis. If the coefficients and the right side of the elliptic equation (4.5) satisfy Hölder conditions, then the second order derivative of every solution also satisfies a Hölder condition [10].

Theorem 4.1. Let B be a solution of (4.5) with boundary conditions $(a)$ and $(b)$ and let $\mathbf{A}$, be calculated from $\mathbf{B}$ by (4.2), then $\mathbf{A}_{s}$ satisfies the hypothesis of Theorem 3.2.

Proof. That $\mathbf{A}_{s}$ is tangent to $S$ and satisfies (3.1) on each face has been proven in Lemma 4.1. Condition $(a)$ of the boundary conditions requires that the component of $\mathbf{A}_{\text {s }}$ tangental to an edge must be continuous across the edge. By Lemma $4.4 \mathbf{A}_{8}$ is continuous differentiable on each face.

Theorem 4.2. If $S$ is a single connected surface the vector potential $\mathbf{A}^{\prime}$ with the tangental projection $\mathbf{A}_{\text {s }}$ is unique in $V$.

Proof. Iet $\mathbf{A}^{\prime \prime}$ be another vector potential satisfying $\nabla \times \mathbf{A}^{\prime \prime}=\mathbf{V}, \nabla \cdot \mathbf{A}^{\prime \prime}=0$, in $V$, and $\mathbf{A}_{T}^{\prime \prime}=\mathbf{A}_{s}$ on $S$. Then

$$
\mathbf{A}^{\prime}-\mathbf{A}^{\prime \prime}=\nabla \gamma
$$

where $\gamma$ is a harmonic function. On $S$

$$
\begin{aligned}
\left(\mathbf{A}^{\prime}-\mathbf{A}^{\prime \prime}\right)_{r} & =\mathbf{A}_{s}-\mathbf{A} . \\
& =0 \\
& =\nabla_{s} \gamma .
\end{aligned}
$$

Since $\nabla_{s} \gamma=0$ on $S, \gamma$ is a constant on $S$. By the maximum principle a harmonic function that is constant on the boundary of a region is constant in the region. Thus, $\nabla \gamma=0$ and $\mathbf{A}^{\prime \prime}=\mathbf{A}^{\prime}$.

Tнеокем 4.3. If $S$ is a sum of multiple disconnected surfaces, and $\mathbf{A}^{\prime}$ is a vector potential such that the net flux of $\mathbf{A}^{\prime}$ on $S_{i}$ is zero, i.e.

$$
\oint_{S_{i}} \mathbf{A}^{\prime} \cdot \mathbf{n} d a=0, \quad i=1, \cdots, N
$$

and has the tangental projection $\mathbf{A}_{\boldsymbol{s}}$ on $S$, then $\mathbf{A}^{\prime}$ is unique in $V$.

Proof. The proof is the same as for Theorem 4.2 to the point where it states that $\gamma$ is constant on $S$. Here we have that $\gamma$ is a constant, $c_{i}$ on $S_{i}, i=1, \cdots, N$, but it may 
be a different constant on each $S_{i}$. The condition that the net flux is zero implies

$$
\begin{aligned}
\int_{s_{i}}\left(\mathbf{A}^{\prime}-\mathbf{A}^{\prime \prime}\right) \cdot \mathbf{n} d a & =\int_{s_{i}} \nabla \boldsymbol{\gamma} \cdot \mathbf{n} d a \\
& =\oint_{S_{i}} \frac{\partial \gamma}{\partial n} d a \\
& =0, \quad i=1, \cdots, N .
\end{aligned}
$$

The Green's identify for $\gamma$ gives

$$
\begin{aligned}
\int_{V}(\nabla \gamma)^{2} d v & =\int_{S} \gamma \frac{\partial \gamma}{\partial n} d a \\
& =\sum_{i=1}^{N} \oint_{S_{i}} C_{i} \frac{\partial \gamma}{\partial n} d a \\
& =\sum_{i=1}^{N} C_{i} \oint_{S_{i}} \frac{\partial \gamma}{\partial n} d a \\
& =0 .
\end{aligned}
$$

Thus, $\nabla \gamma=0$ and $\mathbf{A}^{\prime \prime}=\mathbf{A}^{\prime}$.

5. Boundary condition on the normal component. The boundary condition on the normal component of the vector potential may be expressed as a boundary condition of the third kind by using tensor notation. This boundary condition is found by applying the condition $\nabla \cdot \mathbf{A}^{\prime}=0$ on $S$.

Definition. The Riemann-Christoffel tensor of the surface differentiation of a spatial vector is $R_{i \alpha \beta}^{i}$ where

$$
B_{i, \alpha \beta}-B_{i, \beta \alpha}=R_{i \alpha \beta}^{i} B_{i} .
$$

By performing the covariant differentiating and simplifying $R_{i \alpha \beta}^{i}$ may be expressed as

$$
R_{i \alpha \beta}^{i}=\left(\frac{\partial}{\partial u^{\alpha}}\left\{\begin{array}{c}
j \\
i k
\end{array}\right\}\right) t_{\beta}^{k}-\left(\frac{\partial}{\partial u^{\beta}}\left\{\begin{array}{c}
j \\
i k
\end{array}\right\}\right) t_{\alpha}^{k}+\left\{\begin{array}{c}
l \\
i m
\end{array}\right\}\left\{\begin{array}{l}
j \\
l k
\end{array}\right\}\left(t_{\beta}^{m} t_{\alpha}^{k}-t_{\alpha}^{m} t_{\beta}^{k}\right) .
$$

DEFINITION. $\quad R_{r i \alpha \beta}=g_{r i} R_{i \alpha \beta}^{i}$.

LEMMA 5.1. $R_{r i \alpha \beta}=-R_{i r \alpha \beta}$.

Proof. By using the equations

$$
\begin{aligned}
g_{r j, \alpha} & =0, \\
g_{r j}\left\{\begin{array}{c}
j \\
i k
\end{array}\right\} & =[i k, r]=\partial g_{i r} / \partial x^{k}+\partial g_{r k} / \partial x^{i}-\partial g_{i k} / \partial x^{r}
\end{aligned}
$$

and after some manipulation, we obtain the formula

$$
R_{r i \alpha \beta}=\left(t_{\beta}^{k} \frac{\partial}{\partial u^{\alpha}}-t_{\alpha}^{k} \frac{\partial}{\partial u^{\beta}}\right)\left(\frac{\partial g_{r k}}{\partial x^{i}}-\frac{\partial g_{i k}}{\partial x^{r}}\right)-\left\{\begin{array}{c}
j \\
i k
\end{array}\right\}\left\{\begin{array}{c}
\rho \\
r n
\end{array}\right\} g_{\rho j}\left(t_{\alpha}^{n} t_{\beta}^{k}-t_{\beta}^{n} t_{\alpha}^{k}\right)
$$

from which the antisymmetry is obvious. 
LEMma 5.2. Let B be a vector normal to $S$ and have continuous derivatives of the second order and define an orthogonal coordinate system such that $x^{1}=u^{1}, x^{2}=u^{2}$, and $S$ is the coordinate surface $x^{3}=0$. Then $B_{3, \alpha \beta}=B_{3, \beta \alpha}$.

Proof.

$$
B_{i, \alpha \beta}-B_{i, \beta \alpha}=R_{i \alpha \beta}^{i} B_{i}=g^{r i} R_{r i \alpha \beta} B_{i} .
$$

Since the coordinates are orthogonal

$$
g^{i i}=0, \quad i \neq j .
$$

The only nonzero component of $\mathbf{B}$ is $B_{3}$.

$$
B_{3, \alpha \beta}-B_{3, \beta \alpha}=g^{33} R_{33 \alpha \beta} B_{3}=0
$$

since $R_{33 \alpha \beta}=0$ because of antisymmetry.

LemMA 5.3. Let B be a vector normal to $S$. Then $\nabla . \times \mathrm{B}$ is tangential to $S$ or is zero.

Proof. Since B is normal to $S$

$$
\begin{aligned}
\epsilon_{i j k} n^{i} B^{k} & =0, \\
\left(\epsilon_{i j k} n^{i} B^{k}\right)_{, \alpha} & =\epsilon_{i j k}\left(n_{, \alpha}^{i} B^{k}+n^{i} B_{, \alpha}^{k}\right)=0 .
\end{aligned}
$$

$\nabla . \times \mathbf{B}$ is tangental to $S$ or zero if and only if $\mathbf{n} \cdot \nabla, \times \mathbf{B}=0$.

Writing $\mathbf{n} \cdot \nabla . \times \mathbf{B}$ in tensor notation and substituting in (5.2) we have after some manipulation:

$$
\mathrm{n} \cdot \nabla \cdot \times \mathrm{B}=\epsilon^{\rho \alpha} b_{\alpha \rho} n_{k} B^{k}
$$

$b_{\alpha \rho}$ is a symmetric tensor associated with the second fundamental form of the surface so $\epsilon^{\rho \alpha} b_{\alpha \rho}=0$. Therefore $\mathbf{n} \cdot \nabla . \times \mathbf{B}=0$.

Theorem 5.1. Let B be a vector normal to $S$ with continuous second order derivatives. Then $\nabla \cdot \nabla_{\bullet} \times \mathbf{B}=0$.

Proof. By Lemma 5.3, $\nabla, \times \mathrm{B}$ is tangental to $S$.

$$
\begin{aligned}
(\nabla, \times \mathrm{B})_{\gamma} & =t_{\gamma}^{i}(\nabla, \times \mathrm{B})_{i} \\
& =n_{k} \epsilon_{\gamma \beta} a^{\alpha \beta} g^{m k} B_{m, \alpha}, \\
\nabla . \nabla_{s} \times \mathrm{B} & =a^{\gamma \rho}(\nabla, \times \mathrm{B})_{\gamma, \rho} \\
& =\epsilon_{\gamma \beta} a^{\gamma \rho} a^{\alpha \beta} g^{m k}\left(n_{k, \rho} B_{m, \alpha}+n_{k} B_{m, \alpha \rho}\right) .
\end{aligned}
$$

Let $B_{m}=\left(B_{r} n^{r}\right) n_{m}$

$$
\begin{gathered}
B_{m, \alpha}=\frac{\partial\left(B_{r} n^{r}\right)}{\partial u^{\alpha}} n_{m}+\left(B_{r} n^{r}\right) n_{m, \alpha}, \\
g^{m k} n_{k, \rho} n_{m}=0, \\
g^{m k} n_{k, \rho} n_{m, \alpha}=C_{\rho \alpha}, \\
\epsilon_{\gamma \beta} a^{\gamma \rho} a^{\alpha \beta} C_{\rho \alpha}=\epsilon^{\rho \alpha} C_{\rho \alpha}=0,
\end{gathered}
$$

since $C_{\rho \alpha}$ is symmetric. Thus, 


$$
\begin{aligned}
\nabla \cdot \nabla, \times \mathrm{B} & =\epsilon_{\gamma \beta} a^{\gamma \rho} a^{\alpha \beta} g^{m k} n_{k} B_{m, \alpha \rho} \\
& =\epsilon^{\rho \alpha} n^{m} B_{m, \alpha \rho} .
\end{aligned}
$$

Let the coordinate system be such as that in Lemma 5.2. Then

$$
n^{m} B_{m, \alpha \rho}=n^{3} B_{3, \alpha \rho} \text {. }
$$

By Lemma 5.2 the above expression is symmetric in $\alpha$ and $\rho$. Thus,

$$
\nabla \cdot \nabla \cdot \times \mathbf{B}=\mathbf{0} .
$$

Lemma 5.4. Let A be a spatial vector defined on $S$ and let the coordinate system be the one defined in Lemma 5.2. Then on $S$

$$
\nabla \cdot \mathbf{A}=g^{33} A_{3,3}+\nabla \cdot \cdot \mathbf{A} .
$$

Proof.

$$
\begin{aligned}
& t_{\gamma}^{i}=\delta_{\gamma}^{i}, \quad g^{\alpha \beta}=a^{\alpha \beta} \\
\nabla \cdot \mathbf{A} & =g^{11} A_{1,1}+g^{22} A_{2,2}+g^{33} A_{3,3} \\
& =a^{\alpha \beta} t_{\alpha}^{i} A_{i, \beta}+g^{33} A_{3,3} \\
& =\nabla \cdot \cdot \mathbf{A}+g^{33} A_{3,3}
\end{aligned}
$$

THeOREM 5.2. Let the coordinate system be the one defined in Lemma 5.2 and let the tangential projection of the vector potential be given by (4.2). Then the boundary condition for the normal component of the vector potential may be expressed as

$$
g^{33} \frac{\partial h_{3} A(3)}{\partial x^{3}}-\left(g^{33}\left\{\begin{array}{c}
3 \\
33
\end{array}\right\} h_{3}+2 H\right) A(3)=g^{33}\left\{\begin{array}{l}
\alpha \\
33
\end{array}\right\} h_{\alpha} A(\alpha)
$$

Proof.

$$
\begin{aligned}
\nabla \cdot \mathbf{A} & =\nabla_{\mathfrak{\imath}} \cdot \mathbf{A}+g^{33} A_{3,3}, \\
\nabla \cdot \mathbf{A} & =-2 H A_{(\mathbf{n})}+\nabla_{\bullet} \cdot \mathbf{A}_{\mathbf{\imath}}, \\
\mathbf{A}_{\mathbf{s}} & =\nabla \cdot \times \mathbf{B} .
\end{aligned}
$$

Thus,

$$
\begin{aligned}
\nabla \cdot A_{\mathbf{1}} & =\nabla \cdot \nabla, \times \mathrm{B}=0 \\
A_{3,3} & =\frac{\partial A_{3}}{\partial x^{3}}-\left\{\begin{array}{c}
3 \\
33
\end{array}\right\} A_{3}-\left\{\begin{array}{c}
\alpha \\
33
\end{array}\right\} A_{\alpha}, \\
A_{i} & =h_{i} A(i) \\
A_{(n)} & =A(3), \\
\nabla \cdot \mathbf{A} & =-2 H A(3)+g^{33}\left(\frac{\partial h_{3} A(3)}{\partial x^{3}}-\left\{\begin{array}{c}
3 \\
33
\end{array}\right\} h_{3} A(3)-\left\{\begin{array}{c}
\alpha \\
33
\end{array}\right\} h_{\alpha} A(\alpha)\right)=0 .
\end{aligned}
$$

6. Boundary conditions at a fluid interface. A stationary interface between two viscous fluids or between a viscous fluid and an inviscid fluid will be considered. The boundary condition on the vector potential may be found as previously discussed but 
the vorticity boundary condition can not be calculated from (2.4) since the tangential components of the velocity are not specified on the interface.

A momentum balance on the interface will provide a relation to specify the boundary conditions on the tangential components of the vorticity. Assume that the surface tension is constant and that it is possible to neglect the surface density and the coefficients of dilational and shear surface viscosity. The momentum equation with these assumptions is:

$$
\begin{aligned}
t^{i}-\hat{t}^{i} & =-(2 H \sigma) n^{i}, \\
t^{i} & =T^{i j} n_{i}
\end{aligned}
$$

where $\sigma$ is the surface tension, $t^{i}$ is the stress vector, and $T^{i i}$ is the stress tensor. If $f$ is a function defined on both sides of the interface, denote $f$ as the value on one side of the interface and $\hat{f}$ as the value on the other side. Denote the jump in $f$ as:

$$
f-\hat{f}=[f] .
$$

If the bulk fluid is Newtonian and incompressible, the stress tensor may be expressed as:

$$
T^{i i}=-p g^{i i}+2 \mu D^{i i}
$$

where $D^{i i}$ is the deformation tensor

$$
D^{i j}=\frac{1}{2}\left(g^{i a} V_{, a}^{i}+g^{i a} V_{. a}^{i}\right) .
$$

The vorticity tensor $\Omega^{i i}$ is defined as:

$$
\Omega^{i j}=\frac{1}{2}\left(g^{i q} V_{. a}^{i}-g^{i q} V_{. a}^{i}\right) .
$$

The deformation tensor may be expressed in term of the vorticity tensor as

$$
D^{i j}=\Omega^{i i}+g^{i q} V_{. a}^{i} \text {. }
$$

The scalar product of the vorticity tensor with the normal vector gives:

$$
\Omega^{i j} n_{i}=\frac{1}{2}(\mathrm{~W} \times \mathrm{n})^{i} .
$$

Thus, the stress vector may be expressed as:

$$
t^{i}=-p n^{i}+\mu(\mathrm{W} \times \mathrm{n})^{i}+2 \mu g^{i q} V_{,{ }^{i}}^{i} n_{i} .
$$

Since the equation for the stress vector is linear its difference across the interface may be expressed as the sum of the difference of its individual parts:

$$
\left[t^{i}\right]=-[p] n^{i}+([\mu \mathrm{W}] \times \mathbf{n})^{i}+2 g^{i a} n_{i}\left[\mu V_{. a}^{i}\right] .
$$

The jump in the stress vector may be eliminated by substituting (6.1) into (6.2) and the equation may be rearranged to give

$$
([\mu \mathrm{W}] \times \mathrm{n})^{i}=([p]-2 H \sigma) n^{i}-2 g^{i q} n_{i}\left[\mu V_{. q}^{i}\right]
$$

The cross product of this equation with the normal vector results in

$$
[\mu \mathrm{W}]_{m}-n_{m}(\mathrm{n} \cdot[\mu \mathrm{W}])=-2 \epsilon_{m n i} n^{k} n_{i} g^{i q}\left[\mu V_{, a}^{i}\right] .
$$

Define an orthogonal coordinate system such that the coordinate lines of $x^{1}$ and $x^{2}$ lie in the interface and the coordinate line of $x^{3}$ is normal to the interface. Then 


$$
\begin{gathered}
g^{i j}=0, \quad i \neq j, \\
n_{i}=\left(0,0, g_{33}^{1 / 2}\right) .
\end{gathered}
$$

On the interface $n \cdot \mathrm{V}=0$ so

$$
\begin{gathered}
\partial V^{3} / \partial x^{\alpha}=0, \quad \alpha=1,2 \\
\epsilon_{m n i} n^{n} n_{j} g^{i q}\left[\mu V_{, a}^{i}\right]=\epsilon_{m 3 i}\left(g^{33} \delta_{3}^{i}\left[\mu \frac{\partial V^{3}}{\partial x^{3}-}\right]+g^{i q}\left\{\begin{array}{c}
3 \\
\alpha q
\end{array}\right\}\left[\mu V^{\alpha}\right]\right) .
\end{gathered}
$$

Written in terms of its components (6.3) is

$$
\left[\begin{array}{c}
{\left[\mu W_{1}\right]} \\
{\left[\mu W_{2}\right]} \\
0
\end{array}\right\}=\left[\begin{array}{c}
2 g^{1 / 2} g^{22}\left\{\begin{array}{c}
3 \\
22
\end{array}\right\}\left[\mu V^{2}\right] \\
-2 g^{1 / 2} g^{11}\left\{\begin{array}{c}
3 \\
11
\end{array}\right\}\left[\mu V^{1}\right] \\
0
\end{array}\right\} .
$$

If the interface is between two viscous fluids the normal component of the vorticity is continuous across the interface [11]. If the interface is between a viscous fluid and an inviscid fluid the boundary condition on the normal component of the vorticity may be found from the solenoidal property of the vorticity.

$$
\frac{\partial W^{3}}{\partial x^{3}}+\left\{\begin{array}{c}
i \\
3 i
\end{array}\right\} W^{3}=-\frac{\partial W^{\alpha}}{\partial x^{\alpha}}-\left\{\begin{array}{c}
i \\
\alpha i
\end{array}\right\} W^{\alpha} .
$$

In the special case of a plane interface

$$
W_{1}=W_{2}=\partial W_{3} / \partial x^{3}=0 .
$$

7. Discussion of applications. In the preceding sections it has been shown that in general a second order partial differential equation must be solved to establish the boundary conditions on the vector potential of three-dimensional hydrodynamics. The most general form of this partial differential equation is given by (4.5). Equation (4.2) specifies the tangential components of the vector potential and the boundary condition on the normal component is given by (5.4).

Equations (4.5) and (5.4) reduce to a relatively simple form for the important cases of surfaces which coincide with a coordinate surface. Three of these are given below:

(a) The surface is a plane with $x^{3}=$ constant in Cartesian coordinates.

$$
\begin{gathered}
\partial^{2} B(3) /\left(\partial x^{1}\right)^{2}+\partial^{2} B(3) /\left(\partial x^{2}\right)^{2}=-V(3), \\
\partial A(3) / \partial x^{3}=0 .
\end{gathered}
$$

(b) The surface is spherical in spherical coordinates

$$
\begin{gathered}
\frac{1}{\rho^{2} \sin \theta} \frac{\partial\left(\sin \theta \frac{\partial B(\rho)}{\partial \theta}\right)}{\partial \theta}+\frac{1}{(\rho \sin \theta)^{2}} \frac{\partial^{2} B(\rho)}{\partial \phi^{2}}=-V(\rho), \\
\partial A(\rho) / \partial \rho+(2 / \rho) A(\rho)=0 .
\end{gathered}
$$

(c) The surface is cylindrical in cylindrical coordinates. 


$$
\begin{gathered}
\left(1 / \rho^{2}\right)\left(\partial^{2} B(\rho) / \partial \theta^{2}\right)+\left(\partial^{2} B(\rho) / \partial z^{2}\right)=-V(\rho), \\
(\partial A(\rho) / \partial \rho)+(1 / \rho) A(\rho)=0 .
\end{gathered}
$$

In the particular cases of $\mathrm{n} \cdot \mathrm{V}=0$ on all of $S$ as in the cases studied by Moreau [4] and Hellums and Aziz [5], A, is the zero vector, i.e., $\mathbf{A}^{\prime}$ is normal to $S$.

It is interesting to note that only the normal component of velocity is used to determine the boundary conditions on the vector potential. All components of velocity are used through (2.4) to calculate the boundary values of the vorticity.

Acknowledgment. This work was partially supported by the Public Health Service under Grant HE 09251 and a Predoctoral Fellowship.

\section{ReFERENCES}

[1] H. Lamb, A treatise on the mathematical theory of the motion of fluids, Cambridge University Press, New York, 1879

[2] C. Jacob, Introduction math. à la mécanique des fluids, Gauthier-Villars, Paris, 1959

[3] R. Timman, Memoires offerts a D. Riabouchinsky, Publ. scient. tech. Min. Air, Paris, 1954, 351

[4] J. J. Moreau, Une specification de potential-vecteur en hydrodynamique, Computes Rendus 248, 3406 (1959)

[5] K. Aziz and J. D. Hellums, Numerical solution of the three-dimensional equations of motion for laminar natural convection, Phys. Fluids 10, 314 (1967)

[6] O. D. Kellogg, Foundations of potential theory, Dover, New York, 1953

[7] H. B. Phillips, Vector analysis, Wiley, New York, 1933

[8] G. J. Hirasaki, Ph.D. Thesis, Rice University, Houston, Texas, 1967

[9] R. Aris, Vectors, tensors, and the basic equations of fluid mechanics, Prentice-Hall, Englewood Cliffs, New Jersey, 1962

[10] L. Bers, F. John and M. Schechter, Partial differential equations, Interscience, New York, 1964

[11] C. Truesdell, The classical field theories, Encyclopedia of Physics III, S. Flügge, ed., SpringerVerlag, Berlin, 1960 